\title{
Sangrado de tubo digestivo medio secundario a tumor del estroma gastrointestinal
}

\author{
Small bowel bleeding due to gastrointestinal stromal tumor
}

René Zavala-Gutiérrez*

Palabras clave: Tumores del estroma gastrointestinal, tratamiento quirúrgico.

Keywords: Gastrointestinal stromal tumors, surgical treatment.

${ }^{*}$ Hospital de Alta Especialidad de Veracruz, Secretaría de Salud. México.

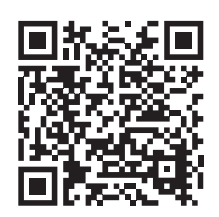

\section{RESUMEN}

Introducción: Los tumores del estroma gastrointestinal son tumores mesenquimales del tracto gastrointestinal que expresan receptores específicos, como la tirosina cinasa c-kit. Representan menos de $1 \%$ de las neoplasias gastrointestinales, la mayoría se localiza en estómago (60$70 \%$ ) e intestino delgado (30\%). Por lo general, presenta sintomatología inespecífica, más frecuentemente sangrado y/o obstrucción intestinal. Presentación de caso: Presentamos un caso de tumor de estroma gastrointestinal con sangrado de tubo digestivo de íleon, en una mujer de 56 años. Conclusión: Su vaga sintomatología lo convierte en un reto diagnóstico. Siendo una patología rara, la sospecha diagnóstica es importante para proporcionar un tratamiento adecuado.

\section{ABSTRACT}

Introduction: Gastrointestinal stromal tumors are mesenchymal tumors of the gastrointestinal tract specifically expressing receptors, such as tyrosine kinase $c$-kit. Represent $<1 \%$ of gastrointestinal neoplasms, the majority is located in the stomach (60-70\%), and the small bowel (30\%). Usually, presents nonspecific symptom, more frequently bleeding and/or intestinal obstruction. Case presentation: We report a case of tumor with digestive tract bleeding in ileum, of a 56 years old female. Conclusion: Its vague symptomatology makes it a diagnostic challenge. Being a rare pathology, diagnostic suspicion is important to provide adequate treatment.

\section{INTRODUCCIÓN}

T os tumores del estroma gastrointestinal $\amalg$ (GIST, por sus siglas en inglés) son definidos como tumores mesenquimales del tracto gastrointestinal que expresan receptores específicos, como tirosina cinasa c-kit (CD117). ${ }^{1}$ Los GIST tienen un patrón indolente de crecimiento que resulta en tumores medianos de diámetro de $8 \mathrm{~cm}$ en el momento del diagnóstico. ${ }^{2}$ Los GIST representan menos de $1 \%$ de las neoplasias gastrointestinales, $20 \%$ de las neoplasias de intestino delgado, con una incidencia de 1020 millones de personas. ${ }^{3}$ La incidencia anual en Estados Unidos es de aproximadamente 4,500 casos al año. ${ }^{4}$ Se localizan la mayoría en estómago (60-70\%), intestino delgado (30\%), colon y esófago $(5 \%)$, pero pueden surgir en cualquier parte del tracto gastrointestinal, desde el esófago hasta el ano. ${ }^{3}$

Recientemente, muchos centros epidemiológicos reportan en sus datos una alta ocurrencia de GIST asociados a otra neoplasia maligna. ${ }^{5}$ La más frecuente asociación es estómago y neoplasia colorrectal. Los reportes señalan rangos de frecuencia de 2.95 a 33\%. ${ }^{6}$

Los GIST se originan de las células intersticiales de Cajal o sus células madre precursoras. Estudios basados en la expresión del protooncogén c-kit apoyan la hipótesis de un carcinogénico común como etiología. ${ }^{7}$

La presentación más común es hemorragia gastrointestinal con o sin obstrucción. Se presenta con síntomas abdominales vagos o sin manifestación clínica específica 69\% de las veces. Suelen ser hallazgos incidentales por

Citar como: Zavala-Gutiérrez R. Sangrado de tubo digestivo medio secundario a tumor del estroma gastrointestinal. Cir Gen. 2021; 43 (1): 51-55. https://dx.doi.org/10.35366/103915 
endoscopia, cirugía, o por imagen en $21 \%$ de los casos y $10 \%$ se encuentran en autopsias. ${ }^{4}$

Se presenta un caso de GIST de intestino delgado en un paciente que manifestaba sangrado intestinal y obstrucción parcial, se le realizó resección quirúrgica con márgenes libres. Esta patología se presenta más frecuentemente como obstrucción, también pueden mostrar sangrado intestinal por la erosión que causa la tumoración; sin embargo, la sintomatología suele ser vaga.

El sangrado es el resultado de la erosión en el tracto gastrointestinal. Otros síntomas resultan del efecto de masa del tumor, que causan disconfort, náusea, emesis y saciedad temprana.

La endoscopia frecuentemente falla para la detección de GIST submucoso y extraluminal, las muestras de biopsia son frecuentemente negativas. La aguja fina de aspiración es guiada por ultrasonido o tomografía, se ha desarrollado como un método para obtención de células tumorales y ha permitido el diagnóstico preoperatorio de GIST por examen histológico con inmunohistoquímica. ${ }^{8}$

El diagnóstico puede ser un reto y consiste en la utilización de endoscopia, ultrasonido, tomografía o resonancia magnética.

La tomografía axial computada (TAC) se recomienda en estos pacientes para diferenciar la causa de obstrucción, la cual puede ayudar a decidir el manejo. La TAC es una recomendación clase II por EAST WORKSHOP para el manejo de la obstrucción de intestino delgado. ${ }^{9}$

Las nuevas masas detectadas clínica o radiológicamente "en pacientes con historia de GIST" deben ser muestreadas por biopsia para exclusión de un GIST no maligno. ${ }^{10}$

El tratamiento consiste en resección quirúrgica en cuña sin linfadenectomía, ésta representa la cura para pacientes con tumor primario localizado. ${ }^{11}$ Ya que los GIST gástricos raramente metastatizan a nódulos linfáticos, éstos no necesitan de linfadenectomía. ${ }^{12}$ Con el fin de lograr una adecuada resección se recomienda un margen libre de $1 \mathrm{a} 2 \mathrm{~cm} .{ }^{13}$

La resección en cuña laparoscópica podría considerarse como procedimiento de elección y una alternativa válida a la cirugía abierta convencional para resección de GIST pequeños de $2 \mathrm{~cm} .{ }^{8}$ El desarrollo de dispositivos de grapado endoscópico y la evidencia de resecciones laparoscópicas de GIST es efectivo con mínima morbilidad y no reporta mortalidad.

Con el avance de la cirugía de mínima invasión, la resección laparoscópica de GIST gástrico de $5 \mathrm{~cm}$ o menos se ha reportado en diversos estudios como factible y segura. La gastrectomía abierta se adoptó usualmente para tumores GIST más grandes en estómago. ${ }^{1}$

La cirugía laparoscópica debe ser considerada en casos de obstrucción intestinal y cáncer. Los resultados son apropiados a corto y largo plazo, siendo similares a los de cirugía abierta. La cirugía laparoscópica tiene corta estancia hospitalaria, menor sangrado y menos requerimiento de medicamento para el dolor. ${ }^{14}$

La invención de mesilato de imatinib abre una nueva perspectiva en el tratamiento de GIST. Especialmente es usado como neoadyuvante en casos con estadios inoperables y para lograr márgenes de resección negativos. Imatinib es un inhibidor competitivo de tirosina cinasa (KIT), el cual ha mostrado ser efectivo para controlar el crecimiento de GIST.

Actualmente, los factores que condicionan un peor pronóstico en los tumores del estroma gastrointestinal, es que su tamaño sea mayor de $5 \mathrm{~cm}$ y que tengan un índice mitótico a 5 mitosis por campo. ${ }^{4,15}$

\section{CASO CLÍNICO}

Mujer de 56 años, la cual consiente la presentación del caso. Con historia de tres meses de evolución, con dolor abdominal, evacuaciones melénicas de escasa cantidad, pérdida de apetito y disminución de peso, acompañado de cefalea, astenia, adinamia, ingresa a urgencias después de presentar lipotimia. La paciente tiene historial quirúrgico de ooforectomía izquierda, colecistectomía laparoscópica, hospitalizaciones previas por sangrado de tubo digestivo bajo y anemia leve; recientemente fue tratada por gastritis crónica. La paciente recibió transfusiones de sangre por hemorragia postcesárea hace 17 años. No toma medicamentos y niega tener alguna alergia.

Al examen físico presenta palidez de tegumentos, abdomen blando, depresible y con distensión, sin dolor a la palpación 
profunda, peristalsis presente y tacto rectal sin sangrado. Tiene anemia severa con nivel de hemoglobina de $2.6 \mathrm{~g} / \mathrm{dl}$, hematocrito $9.1 \%$ y recuento de plaquetas en sangre de $382 \times 10^{3} / \mu \mathrm{l}$. Por lo tanto, se administra una transfusión de sangre.

Se le realiza ultrasonido (USG) abdominal que reporta imagen de tumor en fosa iliaca derecha con medidas de $10.5 \times 5.5 \times 9.9 \mathrm{~cm}$. El estudio de colonoscopia no muestra evidencia de tumor en recto y colon, se observa aparente compresión extrínseca a nivel de orificio de válvula ileocecal (Figura 1).

La tomografía axial computada (TAC) revela tumoración estenosante e infiltrante de las paredes del íleon con lumen estrecho y distensión de asas intestinales (Figuras 2 y 3 ).

Durante la laparotomía exploradora, se localizó tumoración dependiente de íleon terminal de $10 \times 10 \mathrm{~cm}$ con obstrucción parcial de lumen intestinal. Subsecuente se realizó resección e ileostomía abierta, se suministró en el postquirúrgico durante su estancia intrahospitalaria, ceftriaxona de $1 \mathrm{~g}$ y metronidazol de $500 \mathrm{mg}$, durante el procedimiento quirúrgico se cerró muñón con vicryl 3-0 y se envía la pieza quirúrgica a patología (Figura 4).

El estudio de patología reportó resección segmentaria de íleon, con tumor del estroma gastrointestinal (GIST) de $13 \mathrm{~cm}$, con más de 5 mitosis en $50 \mathrm{HPF}$, límites quirúrgicos libres de tumor, hiperplasia reactiva folicular de ganglios

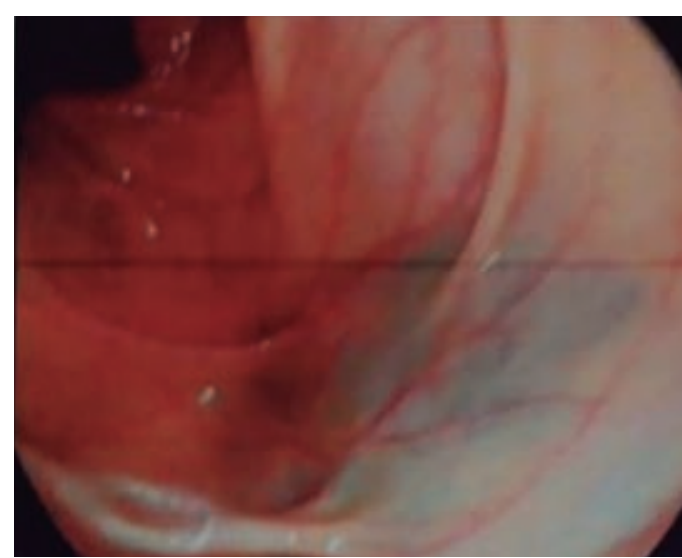

Figura 1: Orificio de válvula ileocecal con aparente compresión extrínseca.

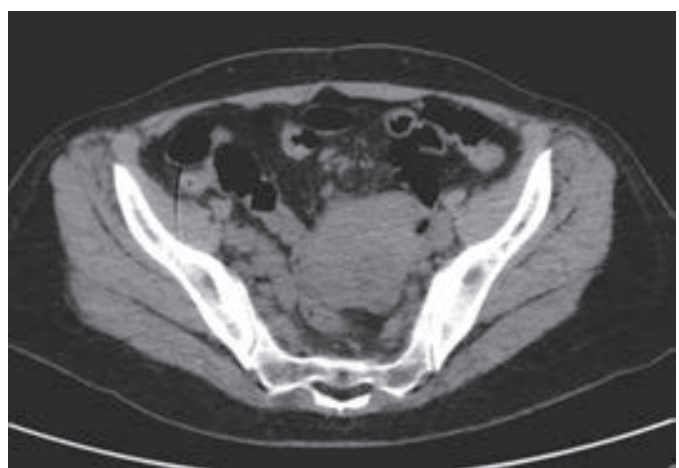

Figura 2: Tomografía simple de abdomen con tumoración abdominal.

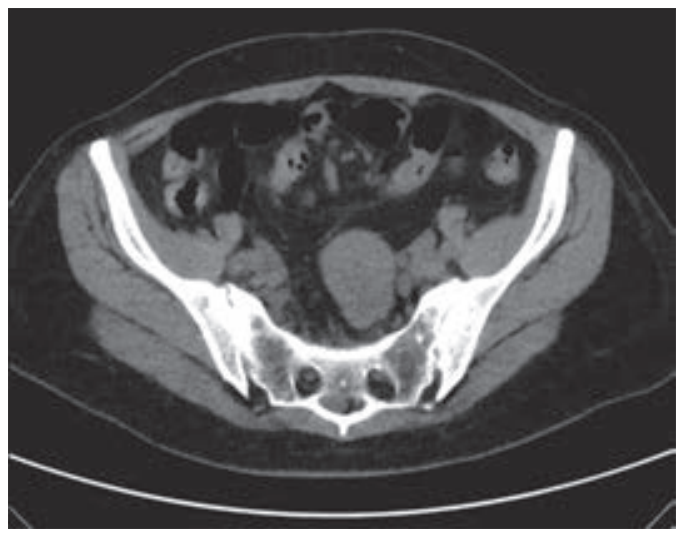

Figura 3: Tomografía axial computada (TAC) con dilatación de asas de intestino delgado, con niveles, sugestivo de obstrucción intestinal.

linfáticos. La paciente al recuperarse es dada de alta en su tercer día postoperatorio.

\section{RESULTADO}

La paciente no presentó complicaciones posteriores a la cirugía, encontrándose asintomática y estable al momento de su alta. Después fue enviada para continuar con su manejo por la consulta de oncología médica para su seguimiento.

\section{DISCUSIÓN}

Este caso presentaba una obstrucción parcial y sintomatología vaga, cerca de $70 \%$ de GIST son sintomáticos en el momento del diagnóstico.

Un pequeño porcentaje requiere cirugía de urgencia; en este caso, la paciente presentó 

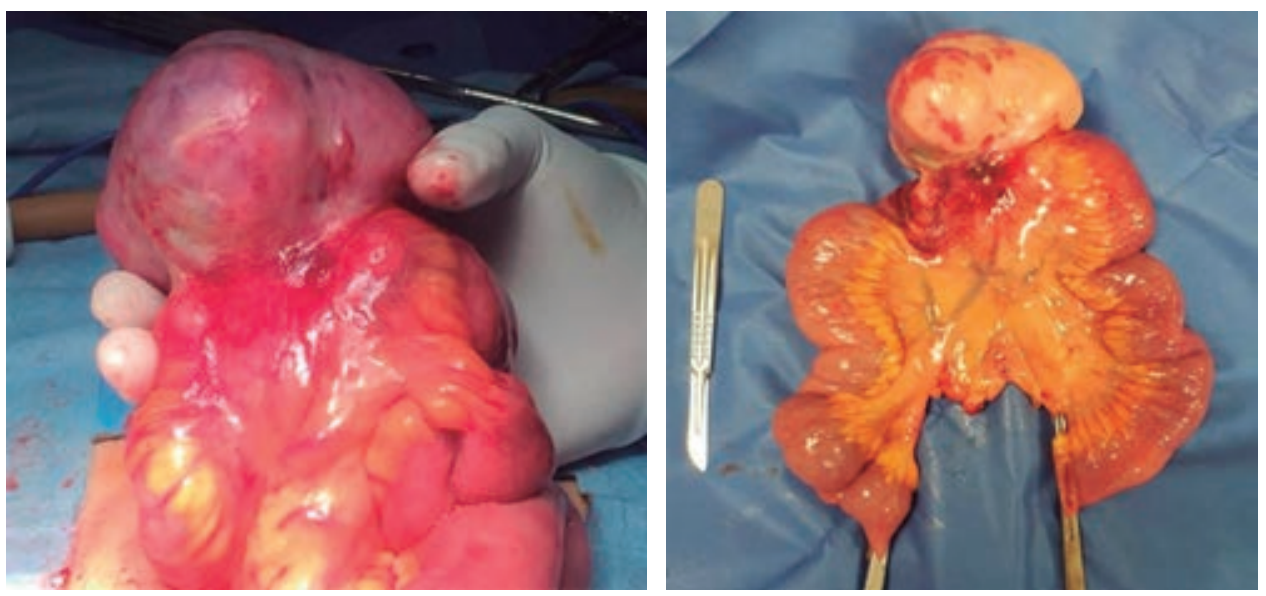

Figura 4: Tumor del estroma gastrointestinal de ileon de $10 \times 10 \mathrm{~cm}$.

una obstrucción parcial y sangrado crónico, requiriendo mejorar condiciones con transfusiones, presentó obstrucción, puede resultar en diferentes características de estos tumores: crecimiento continuo de la lesión que causa oclusión directa del intestino, como en este caso, siendo la intususcepción relativamente rara y sólo se reporta en pocos casos en la literatura. ${ }^{16}$

Para su diagnóstico se utilizó TAC, la cual se recomienda, ya que fue posible diferenciar la causa de obstrucción.

Fue posible un tratamiento curativo, ya que se realizó la resección completa, evitando la ruptura del tumor y con márgenes macroscópicos negativos, siendo su manejo potencialmente curativo.

Aunque hubiera sido preferible la resección por técnica laparoscópica por su baja morbilidad y mortalidad, la paciente presentó una buena evolución.

El tamaño del tumor representa un factor pronóstico negativo, y la resección con márgenes libres es curativa, siendo para la paciente predictores de buen pronóstico. ${ }^{17}$

\section{CONCLUSIÓN}

El GIST es una patología poco frecuente; sin embargo, se puede llegar a requerir manejo médico de urgencia. En pacientes con síntomas de sangrado y/u obstrucción, se debe sospechar la presencia de GIST al encontrar tumoraciones durante estudios de imagen.
Es de gran utilidad la realización de TAC, como se presentó en el caso, para poder establecer el diagnóstico. Se realizó la resección de la pieza de patología con adecuados márgenes libres, mediante cirugía abierta.

En casos de tumores localizados, en los que es posible su resección completa, es preferible la utilización de técnica laparoscópica por su baja morbilidad y mortalidad.

\section{REFERENCIAS}

1. Hsiao CY, Yang CY, Lai IR, Chen CN, Lin MT. Laparoscopic resection for large gastric gastrointestinal stromal tumor (GIST): intermediate follow-up results. Surg Endosc. 2015; 29: 868-873.

2. Hirota S, Isozaki K, Moriyama Y, Hashimoto K, Nishida $\mathrm{T}$, Ishiguro S, et al. Gain-of-function mutations of c-kit in human gastrointestinal stromal tumors. Science. 1998; 279: 577-580.

3. Stamatakos M, Douzinas E, Stefanaki C, Safioleas $P$, Polyzou E, Levidou G, et al. Gastrointestinal stromal tumor. World J Surg Oncol. 2009; 7: 61.

4. Liegl-Atzwanger B, Fletcher JA, Fletcher CD. Gastrointestinal stromal tumors. Virchows Arch. 2010; 456: 111-127.

5. Stratakis CA, Carney JA. The triad of paragangliomas, gastric stromal tumours and pulmonary chondromas (Carney triad), and the dyad of paragangliomas and gastric stromal sarcomas (Carney-Stratakis syndrome): molecular genetics and clinical implications. J Intern Med. 2009; 266: 43-52.

6. Agaimy A, Wunsch PH, Sobin LH, Lasota J, Miettinen M. Occurrence of other malignancies in patients with gastrointestinal stromal tumors. Semin Diagn Pathol. 2006; 23: 120-129.

7. Gopal SV, Langcake ME, Johnston E, Salisbury EL. Synchronous association of small bowel stromal tumour with colonic adenocarcinoma. ANZ J Surg. 2008; 78: 827-828. 
8. Basu S, Balaji S, Bennett DH, Davies N. Gastrointestinal stromal tumors (GIST) and laparoscopic resection. Surg Endosc. 2007; 21: 1685-1689.

9. Diaz JJ Jr, Bokhari F, Mowery NT, Acosta JA, Block EF, Bromberg WJ, et al. Guidelines for management of small bowel obstruction. J Trauma. 2008; 64: 1651-1664.

10. Hechtman JF, DeMatteo R, Nafa K, Chi P, Arcila ME, Dogan S, et al. Additional primary malignancies in patients with gastrointestinal stromal tumor (GIST): a clinicopathologic study of 260 patients with molecular analysis and review of the literature. Ann Surg Oncol. 2015; 22: 2633-2639.

11. Heinrich MC, Corless CL. Gastric Gl stromal tumors (GISTs): the role of surgery in the era of targeted therapy. J Surg Oncol. 2005; 90: 195-207.

12. Matthews BD, Walsh RM, Kercher KW, Sing RF, Pratt BL, Answini GA, et al. Laparoscopic vs open resection of gastric stromal tumors. Surg Endosc. 2002; 16: 803-807.

13. Rosen MJ, Heniford BT. Endoluminal gastric surgery: the modern era of minimally invasive surgery. Surg Clin North Am. 2005; 85: 989-1007.

14. Oida Y, Motojuku M, Morikawa G, Mukai M, Shimizu $\mathrm{K}$, Imaizumi T, et al. Laparoscopic-assisted resection of gastrointestinal stromal tumor in small intestine. Hepatogastroenterology. 2008; 55: 146-149.

15. Schneider-Stock R, Boltze C, Lasota J, Peters B, Corless $\mathrm{CL}$, Ruemmele P, et al. Loss of p16 protein defines high-risk patients with gastrointestinal stromal tumors: a tissue microarray study. Clin Cancer Res. 2005; 11: 638-645.

16. Fischer C, Nagel H, Metzger J. Image of the month. Gastrointestinal stromal tumor of the small bowel. Arch Surg. 2009; 144: 379-380.

17. Everett M, Gutman H. Surgical management of gastrointestinal stromal tumors: analysis of outcome with respect to surgical margins and technique. J Surg Oncol. 2008; 98: 588-593.

Consideraciones y responsabilidad ética: El autor declara que los procedimientos seguidos se conformaron a las normas de ética. En este artículo no aparecen datos de pacientes.

Financiamiento: El autor declara que no existe financiamiento.

Conflicto de intereses: El autor declara que no existe ningún conflicto de intereses en la realización del trabajo.

Correspondencia:

René Zavala-Gutiérrez

E-mail: rene8may@hotmail.com 